\title{
A Multi-Well Thin-Si LAPS and All-in-One Readout System for Ion Activity Monitor of Epithelium Cells ${ }^{\dagger}$
}

\author{
Yu-Jing Lin ${ }^{1}$, Po-Yen Lin ${ }^{1}$, Yu-Ping Chen ${ }^{1}$, Hui-Ling Liu ${ }^{2}$, Tsung-Ru Wu ${ }^{3}$, Hsin-Chih Lai ${ }^{3}$, \\ Wei-Chun Chin ${ }^{4}$, Chih-Hong Lo ${ }^{5}$, Tsann-Long Hwang ${ }^{5}$, Chia-Ming Yang 1,2,5,* and \\ Chao-Sung Lai ${ }^{2,6,7}$ \\ 1 Institute of Electro-Optical Engineering, Chang Gung University, Taoyuan 333, Taiwan; \\ wendy326446@gmail.com (Y.-J.L.); miraculous0218@hotmail.com (P.-Y.L.); \\ enjoytw8555@gmail.com (Y.-P.C.) \\ 2 Department of Electronic Engineering, Chang Gung University, Taoyuan 333, Taiwan; \\ hll20121219@gmail.com (H.-L.L.); cslai@mail.cgu.edu.tw (C.-S.L.) \\ 3 Department of Medical Biotechnology and Laboratory Science, Chang Gung University, Taoyuan 333, \\ Taiwan; purpleeri@gmail.com (T.-R.W.); hclai@mail.cgu.edu.tw (H.-C.L.) \\ 4 Bioengineering Department, University of California, Merced, CA 95343, USA; wchin2@ucmerced.edu \\ 5 Department of General Surgery, Chang Gung Memorial Hospital at Linkou, Taoyuan 333, Taiwan; \\ albertloch@gmail.com (C.-H.L.); hwangtl@cloud.cgmh.org.tw (T.-L.H.) \\ 6 Department of Nephrology, Chang Gung Memorial Hospital at Linkou, Taoyuan 333, Taiwan \\ 7 Department of Materials Engineering, Ming Chi University of Technology, New Taipei City 24301, Taiwan \\ * Correspondence: cmyang@mail.cgu.edu.tw; Tel.: +886-3-211-8800 (ext. 5960) \\ + Presented at the Eurosensors 2018 Conference, Graz, Austria, 9-12 September 2018.
}

Published: 19 November 2018

\begin{abstract}
To measure the ion activities of cells, an easy-access and fully-integrated system is necessary in culture room with high cleanness and easy maintenance. A new sensor structure integrated with readout system based on light-addressable potentiometric sensor (LAPS) to quantitatively monitor real-time cell activity with the advantages of label free and 2D image ability is proposed. The difference of cell number and acidification could be easily observed by 2D images by means of this proposed methodology.
\end{abstract}

Keywords: 2D image; cell culture; LAPS

\section{Introduction}

The ion activities of cells are important in cell interactions with drugs and toxicity [1]. An easyaccess and fully-integrated system is necessary to establish in culture room with high cleanness and easy maintenance. Compare to the ability of conventional microscope, the ion activity of living cells should be also monitored [2]. Light-addressable potentiometric sensor (LAPS) could be a potential solution to provide this possibility with advantages of biosensor abilities [3]. Many LAPS scanning measurement systems were proposed including DLP [4], OLED [5], multi light fibers [6] and analog micro mirror [7]. Until now, no commercial instrument is available. We proposed a new device structure integrated with readout system based on LAPS to quantitatively monitor real-time cell activity with the advantages of label free and 2D image ability.

\section{Materials and Methods}

A new LAPS device structure is fabricated as shown in schematic and SEM picture in Figure 1a,b by the detail process flow shown in previous literature [8]. A double-side polished P-type silicon wafer is started with thickness of $300 \mu \mathrm{m}$. Thermal growth $\mathrm{SiO}_{2}$ and $\mathrm{Si}_{3} \mathrm{~N}_{4}$ by low-pressure chemical 
vapor deposition (LPCVD) both with thickness of $50 \mathrm{~nm}$ were deposited on the both side of Si wafer. Then back side of $\mathrm{Si}$ was selectively etched by a sequence of dry and wet etching to the thickness of $90 \mu \mathrm{m}$ by the patterns transferred from standard photolithography process. $\mathrm{Si}_{3} \mathrm{~N}_{4}$ layers are used as the sensing membrane on the top side and the hard mask layer on the back side during $\mathrm{KOH}$ wet etching. To test the multi-well monitor, a special PDMS container is fabricated as the design shown in Figure 1c. Finally, this container could be fixed on $\mathrm{Si}_{3} \mathrm{~N}_{4}$ surface of LAPS after $\mathrm{O}_{2}$ plasma treatment. 4 wells in the bottom layer and 1 big well in the top layer are used for cell culture and for LAPS measurement, respectively. An epithelium cell line in proximal tubule of pig's kidney, LLCPK1, is cultured directly on LAPS. As shown in Figure 2, a portable system of FPGA-based LAPS embedded with self-designed incubator is used to perform experiments. Temperature and humidity could be controlled by PID controller, which is similar to commercial incubator. To avoid contamination during cell culture, FPGA-based LAPS system is fully sealed and fixed in this self-design incubator.

(a)

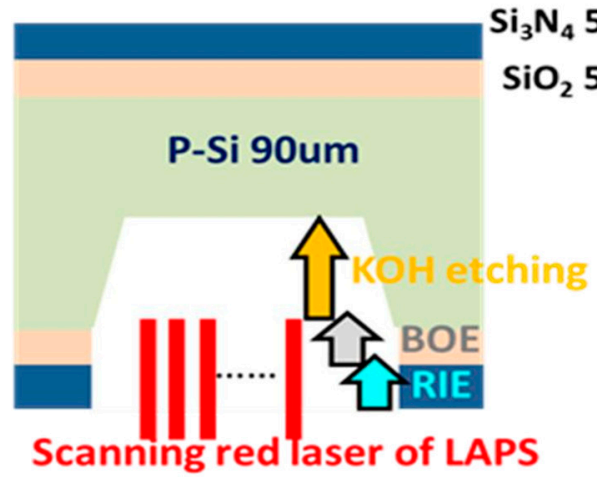

(c)

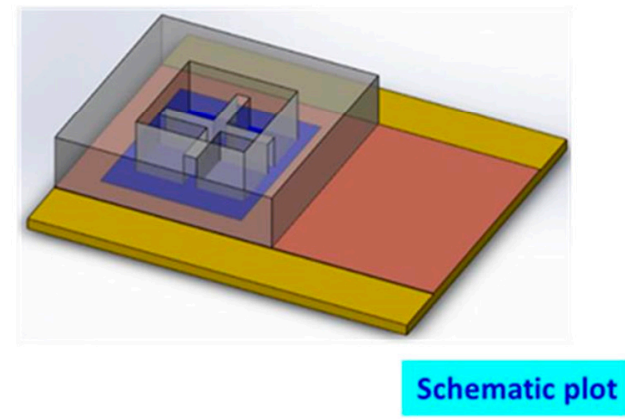

(b)

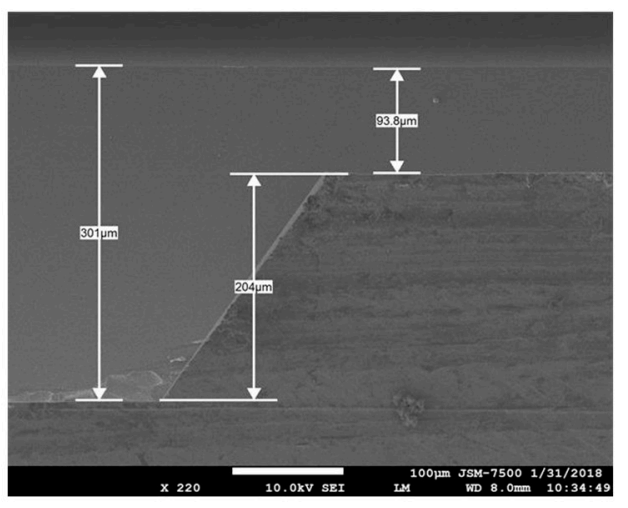

(d)

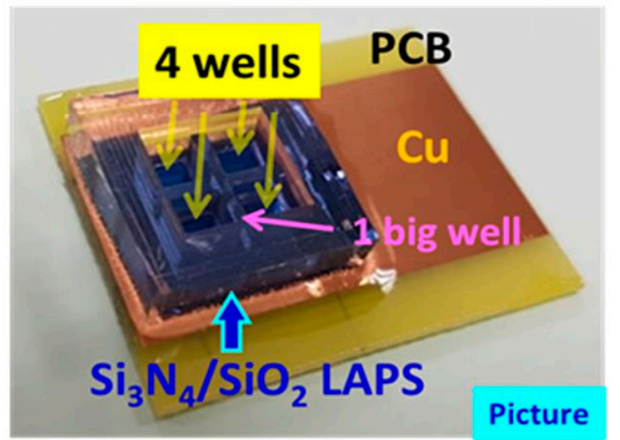

Figure 1. (a) Cross-sectional schematic plot, (b) SEM picture, (c) schematic 3D plot and (d) picture of real LAPS sample.

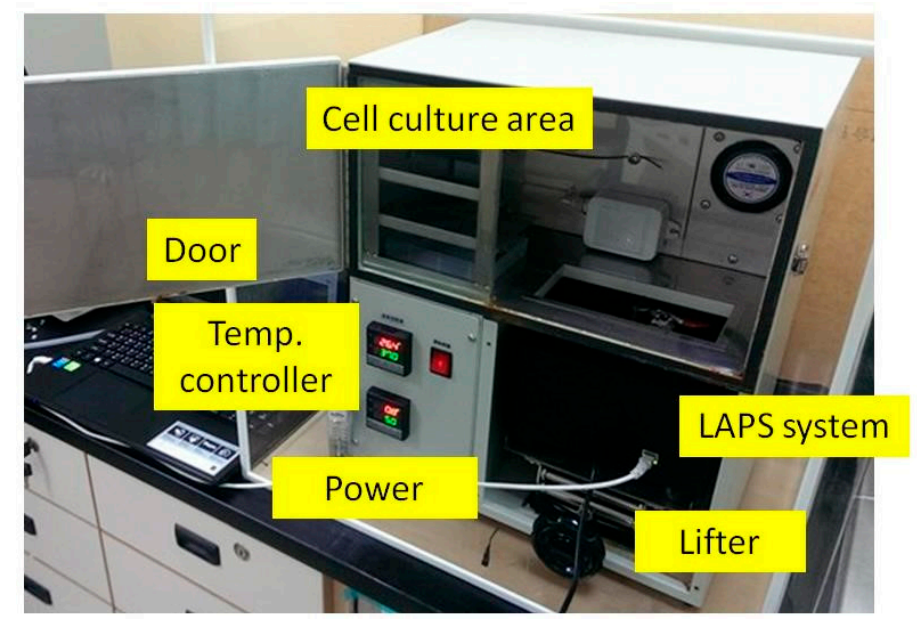

Figure 2. Self-designed incubator to embedded LAPS measurement system and sensor chip. 


\section{Results \& Discussion}

In Figure 3a, OM pictures of 4 wells with different cell concentration are shown. Culture status quite matched the cell numbers in the seeding process. Cell acidification could be easily checked by the color of measured photocurrent by FPGA-based LAPS. More cell growth induces more negative charges and less photocurrent as shown in blue color as shown in Figure $3 b$. Then more acidification after changed medium to HBSS for $30 \mathrm{~min}$ can be observed with a high photocurrent presented in red color as shown in Figure 3c. This developed device and readout system is proven to monitor the ion activity of epithelium cells easily and efficiently

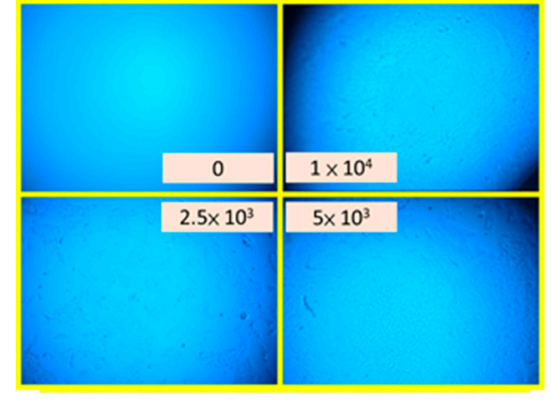

(a) OM images

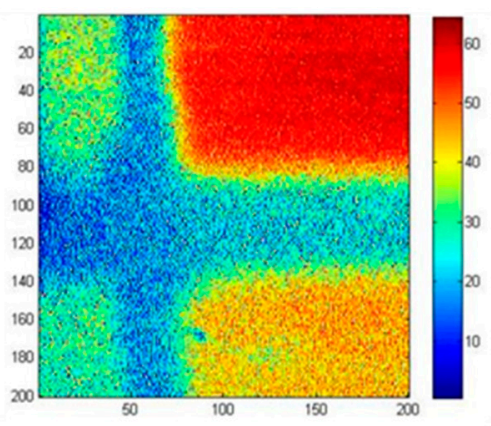

Culture 24h: (b) acidification $0 \mathrm{~min}$

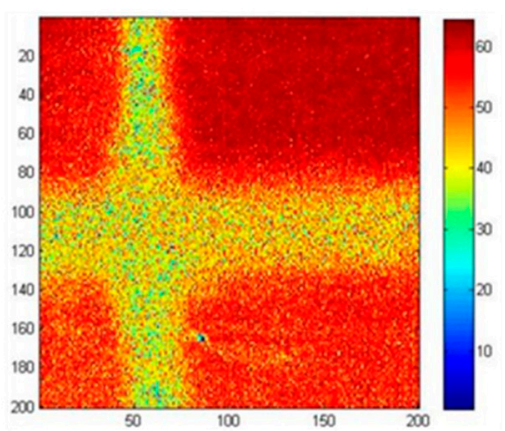

(c) acidification $30 \mathrm{~min}$

Figure 3. (a) OM images, (b) cultured for $24 \mathrm{~h}$ and (c) acidification for $30 \mathrm{~min}$ in HBSS solution of cell directly culture on Si3N4 LAPS surface with different cell number.

\section{Conclusions}

With the proper device fabrication and measurement system embedded with a self-designed incubator, the success rate of directly cell cultured LAPS chip is clearly increased. In current setting, photocurrent decreases with cell numbers due to the negative charges provided by cells. However, cell acidification could be also observed by changing medium to HBSS for $30 \mathrm{~min}$. More cells make more acidification and following higher photocurrent. The platform could be applied in the cell relative ion activity and mechanism study.

Author Contributions: T.-L.H., C.-M.Y., and C.-S.L. conceived and designed the experiments; Y.-J.L., P.-Y.L., Y.P.C. performed the experiments; W.-C.C., C.-H.L., T.-L.H., C.-M.Y., and C.-S.L. analyzed the data; T.-R.W. and H.-C.L. contributed reagents/materials/analysis tools; C.-M.Y. wrote the paper.

Acknowledgments: This research was partially supported by the Ministry of Science and Technology of the Republic of China under Contract no. MOST 105-2221-E-182-057-MY3.

Conflicts of Interest: The authors declare no conflict of interest.

\section{References}

1. Takahashi, A.; Camacho, P.; Lechleiter, J.D.; Herman, B. Measurement of Intracellular Calcium. Phys. Rev. 1999, 79, 1089-1125.

2. Shaibani, P.M.; Etayash, H.; Naicker, S.; Kaur, K.; Thundat, T. Metabolic study of cancer cells using a pH sensitive hydrogel nanofiber light addressable potentiometric sensor. ACS Sens. 2017, 2, 151-156.

3. Gu, Y.; Ju, C.; Li, Y.; Shang, Z.; Wu, Y.; Jia, Y.; Niu, Y. Detection of circulating tumor cells in prostate cancer based on carboxylated graphene oxide modified light addressable potentiometric sensor. Biosens. Bioelectron. 2015, 66, 24-31.

4. Wagner, T.; Werner, C.F.; Miyamotoa, K.; Schöning, M.J.; Yoshinobu, T. Development and characterisa tion of a compact light-addressable potentiometric sensor (LAPS) based on the digital light processi ng (DLP) technology for flexible chemical imaging. Sens. Actuators B 2012, 170, 34-39.

5. Werner, C.F.; Wagner, T.; Miyamotoc, K.; Yoshinobuc, T.; Schöning, M.J. High speed and high resolut ion chemical imaging based on a new type of OLED-LAPS set-up. Sens. Actuators B 2012, 175, 118-122. 
6. Miyamotoa, K.; Itabashi, A.; Wagner, T.; Schöning, M.J.; Yoshinobu, T. High-speed chemical imagingi nside a microfluidic channel. Sens. Actuators B 2014, 194, 521-527.

7. Das, A.; Yang, C.-M.; Chen, T.-C.; Lai, C.-S. Analog micromirror-LAPS for chemical imaging and zoom-in application. Vacuum 2015, 118, 161-166.

8. Yang, C.-M.; Zeng, W.-Y.; Chen, Y.-P.; Chen, T.-C. Surface modification for high photocurrent and pH sensitivity in a silicon-based light-addressable potentiometric sensor. IEEE Sens. J. 2018, 18, 2253-2259.

(C) 2018 by the authors; Licensee MDPI, Basel, Switzerland. This article is an open access article distributed under the terms and conditions of the Creative Commons Attribution (CC BY) license (http://creativecommons.org/licenses/by/4.0/). 